\title{
Optical luminosity functions of the Abell galaxy cluster ABCG 209 at $z=0.21^{\star}$
}

\author{
A. Mercurio ${ }^{1}$, M. Massarotti ${ }^{2}$, P. Merluzzi ${ }^{2}$, M. Girardi ${ }^{1}$, F. La Barbera ${ }^{2}$, and G. Busarello ${ }^{2}$ \\ 1 Dipartimento di Astronomia, Università degli Studi di Trieste, via Tiepolo 11, 34100 Trieste, Italy \\ e-mail: mercurio@ts.astro.it; girardi@ts.astro.it \\ 2 INAF - Osservatorio Astronomico di Capodimonte, via Moiariello 16, 80131 Napoli, Italy
}

Received 27 February 2003 / Accepted 19 June 2003

\begin{abstract}
We derive the luminosity functions in three bands $(B V R)$ for the rich galaxy cluster ABCG 209 at $z=0.21$. The data cover an area of $\sim 78 \operatorname{arcmin}^{2}$ in the $B$ and $R$ bands, while a mosaic of three pointings was obtained in the $V$ band, covering an area of approximately $160 \mathrm{arcmin}^{2}$. The galaxy sample is complete to $B=22.8\left(N_{\text {gal }}=339\right), V=22.5\left(N_{\text {gal }}=1078\right)$ and $R=22.0\left(N_{\text {gal }}=679\right)$. Although the fit of a single Schechter function cannot be rejected in any band, the luminosity functions are better described by a sum of two Schechter functions for bright and faint galaxies, respectively. There is an indication for a presence of a dip in the luminosity functions in the range $V=20.5-21.5$ and $R=20.0-21.0$. We find a marked luminosity segregation, in the sense that the number ratio of bright-to-faint galaxies decreases by a factor 4 from the center to outer regions. Our analysis supports the idea that ABCG 209 is an evolved cluster, resulting from the merger of two or more sub-clusters.
\end{abstract}

Key words. galaxies: clusters: individual: Abell 209 - galaxies: photometry - galaxies: luminosity function, mass function

\section{Introduction}

Galaxy luminosity function (LF) is a powerful tool to constrain galaxy formation and evolution, since it is directly related to the galaxy mass function and hence to the spectrum of initial perturbations. Hierarchical clustering models predict a mass distribution characterised by a cut-off above a given mass $M^{*}$ and well described by a power law at low masses (Press \& Schechter 1974). Starting from these results Schechter (1976) analysed the luminosity distribution of 14 galaxy clusters observed by Oelmer (1974), by introducing an analytical description in the form:

$\phi(L) \mathrm{d} L=\phi^{*}\left(\frac{L}{L^{*}}\right)^{\alpha} \mathrm{e}^{-\left(\frac{L}{L^{*}}\right)} \mathrm{d}\left(\frac{L}{L^{*}}\right)$.

In the Schechter function $\phi^{*}$ is a normalization density and the shape of the LF is described by $L^{*}$, a characteristic cut-off luminosity, and $\alpha$, the faint-end slope of the distribution. He used this function to describe the global LF of all galaxy types and suggested the value $\alpha=-5 / 4$.

Although investigated in several works, the universality of the LF faint-end slope is still controversial. The value of the faint-end slope turns out to be $\alpha \sim-1$ for field galaxies (e.g., Efstathiou et al. 1988; Loveday et al. 1992), while clusters

Send offprint requests to: A. Mercurio,

e-mail: mercurio@ts.astro.it

* Based on observations collected at the European Southern Observatory, Chile (Proposal ESO NM-0 68.A-0116). and groups seem to have steeper slopes, $-1.8<\alpha<-1.3$ (e.g., De Propris et al. 1995; Lumsden et al. 1997; Valotto et al. 1997), suggesting the presence of a larger number of dwarf galaxies (but see also e.g., Lugger 1986; Colless 1989; Trentham 1998). Changes in the slope of the faint-end of the LF in clusters can be related to environmental effects. An increase of the steepening of the LF faint-end in the cluster outer regions was actually observed (Andreon 2001) and explained taking into account that the various dynamical processes which can destroy dwarf galaxies act preferentially in the higherdensity cores.

Lopez-Cruz et al. (1997) showed that clusters with a flat LF $(\alpha \sim-1)$ are a homogeneous class of rich clusters with a single dominant galaxy, symmetrical single peaked X-ray emission and high gas masses. Irregular clusters have a steeper faint-end, in particular, the LFs of ABCG 1569 and Coma which present substructures, can be suitably fitted with the sum of two Schechter functions with $\alpha=-1$ and $\alpha \geq-1.4$ (Lopez-Cruz et al. 1997). Trentham (1997) also suggested that the faint-end slope of the LF flattens as clusters evolve because of the destruction of dwarf galaxies by merging with giants galaxies.

The density of the environment seems to affect the distribution of galaxy luminosity in the sense that Schechter fits are poor for galaxies in dense environment, where there is an indication of a dip (e.g., Driver et al. 1994; Biviano et al. 1995; Wilson et al. 1997; Molinari et al. 1998; Garilli et al. 1999; 
Näslund et al. 2000; Yagi et al. 2002). Trentham \& Hodgking (2002) identified two types of galaxy LF, one for dynamically evolved regions (i.e. region with a high elliptical galaxy fraction, a high galaxy density, and a short crossing time), such as Virgo cluster and Coma cluster, and one for unevolved regions, such as the Ursa Major cluster and the Local Group. A dip is present in the LF of Virgo and Coma and is absent in LFs of Ursa Major and Local Group.

The differences in shape of the LFs from cluster to cluster could be explained assuming that the total LF is the sum of type specific luminosity functions (hereafter TSLFs), each with its universal shape for a specific type of galaxies (Binggeli et al. 1988). The total LF then assumes a final shape which can be different from cluster to cluster according to the mixture of different galaxy types. Therefore, different mixtures of galaxies, induced by cluster-related processes, may be at the origin of the presence and of the different shape of dips seen in cluster LFs and may be responsible for the differences seen in the total LFs among field, groups and galaxy clusters.

Indeed, dips are found in several clusters, occurring roughly at the same absolute magnitude $\left(M_{\mathrm{R} \text {,dip }} \sim-19.4\right.$ or $\left.\sim M^{*}+2.5\right)$, within a range of about one magnitude, suggesting that clusters have comparable galaxy population. However the dips may have different shapes, and also depend on the cluster region. This could be related to the relative abundances of galaxy types, which depend on the global properties of each cluster and on the local density (Durret et al. 1999).

In this work we study the Abell galaxy cluster ABCG 209 at $z=0.21$ (Kristian et al. 1978; Wilkinson \& Oke 1978; Fetisova 1981; Mercurio et al. 2003, hereafter Paper I) which is a rich, $\mathrm{X}$-ray luminous, and massive cluster (richness class $R=$ 3, Abell et al. 1989; $L_{\mathrm{X}}(0.1-2.4 \mathrm{keV}) \sim 14 \times 10^{44} h_{50}^{-2} \mathrm{erg} \mathrm{s}^{-1}$, Ebeling et al. 1996; $T_{\mathrm{X}} \sim 10 \mathrm{keV}$, Rizza et al. 1998; $M\left(<R_{\text {vir }}\right)=1.6-2.2 \times 10^{15} h_{100}^{-1} M_{\odot}$, Paper I).

The cluster shows an elongation and asymmetry in the X-ray emission with two main clumps (Rizza et al. 1998), but no strong cooling flow is detected. The dynamical analysis presented in Paper I showed that ABCG 209 is characterized by a very high value of the line of sight velocity dispersion: $\sigma_{\mathrm{v}}=1250-1400 \mathrm{~km} \mathrm{~s}^{-1}$ and by a preferential SE-NW direction as indicated by: a) the presence of a velocity gradient in the velocity field; b) the elongation in the spatial distribution of colour-selected cluster members; c) the elongation of the X-ray contour levels in the Chandra image; d) the elongation of the $\mathrm{cD}$ galaxy. There is significant evidence of substructure, as shown by the Dressler \& Schectman test. The two-dimensional distribution of the colour-selected members shows a strong luminosity segregation. Furthermore, the young dynamical state is also indicated by the possible presence of a radio halo (Giovannini et al. 1999), possibly a remnant of a recent cluster merger (Feretti 2002).

This observational scenario suggests that ABCG 209 is presently undergoing strong dynamical evolution with the merging of two or more sub-clumps along the SE-NW direction, but could not allow us to discriminate between two alternative pictures (Paper I). The merging might be either in a very early dynamical state, where the clumps are still in a premerging phase, or in a more advanced state, where luminous galaxies trace the remnant of the core-halo structure of a premerging clump hosting the $\mathrm{cD}$ galaxy.

In order to further investigate the cluster dynamical state and to discriminate between the previous pictures, we derived the LFs by using new photometric data for ABCG 209 based on ESO-NTT imaging in the $B, V$ and $R$ wavebands. The new photometric data are presented in Sect. 2. In Sect. 3 we describe the data reduction, and the photometric calibrations. The aperture photometry is presented in Sect. 4, whereas Sect. 5 deals with the LFs and Sect. 6 with the spatial distribution of galaxies of different luminosity. Section 7 is dedicated to the summary and the discussion of the results. In this work we assume $H_{0}=70 \mathrm{~km} \mathrm{~s}^{-1} \mathrm{Mpc}, \Omega_{\mathrm{m}}=0.3, \Omega_{\Lambda}=0.7$. According to this cosmology, 1 arcmin corresponds to $0.205 \mathrm{Mpc}$ at $z=0.209$.

\section{Observations}

New observations of the galaxy cluster ABCG 209 were carried out at the ESO New Technology Telescope (NTT) with the EMMI instrument in October 2001. The data include $B-$, $V$ - and $R$-band imaging, plus multi-slit spectroscopy (EMMINTT) for 112 cluster members. The spectroscopic data are presented in Paper I.

A field of $9.2^{\prime} \times 8.6^{\prime}\left(1.9 \times 1.8 h_{70}^{-2} \mathrm{Mpc}^{2}\right)$, was observed in the $B, V$ and $R$ bands around the cluster center. In order to sample the cluster at large distance from the center, we observed other two fields in $V$ band. The total observed area in $V$ band, accounting for overlapping, is $\sim 160 \operatorname{arcmin}^{2}$ and is shown in Fig. 1. The relevant information on the photometry are summarized in Table 1. Standard stars from Landolt (1992) were also observed before and after the scientific exposures, and were used for the photometric calibration.

\section{Data reduction and photometric calibration}

Standard procedures were employed for bias subtraction, flatfield correction, and cosmic ray rejection using the $\operatorname{IRAF}^{1}$ package. For each waveband the flat-field was obtained by combining twilight sky exposures. After bias subtraction and flat-field correction the images were combined using the IRAF task IMCOMBINE with the CRREJECT algorithm. Residual cosmic rays and hot pixels were interpolated applying the IRAF task COSMICRAYS. The resulting images show a uniform background with typical $\mathrm{rms}$ of $2.3 \%, 1.4 \%$, and $1.1 \%$ for the $B, V$, and $R$ bands, respectively.

The photometric calibration was performed into the Johnson-Kron-Cousins photometric system by using the Landolt standard fields. The instrumental magnitudes of the stars were measured in a fixed aperture by using the IRAF packages APPHOT and DAOPHOT. The aperture size was chosen in order to i) enclose the total flux, ii) obtain the maximum signal-to-noise ratio. By comparing the magnitudes of the stars in different apertures, we found that a reasonable

\footnotetext{
${ }^{1}$ IRAF is distributed by the National Optical Astronomy Observatories, which are operated by the Association of Universities for Research in Astronomy, Inc., under cooperative agreement with the National Science Foundation.
} 


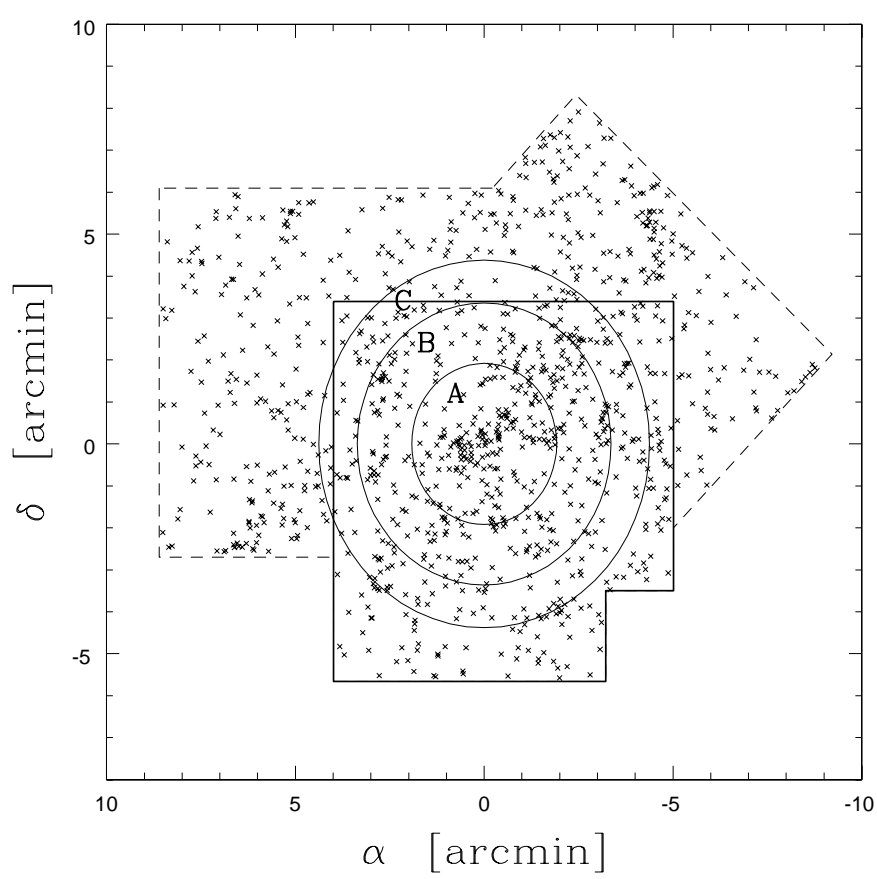

Fig. 1. Area covered by the photometry in the region of ABCG 209 . All the galaxies brighter than $V=21.5$ are marked with crosses. The central field (solid contour) was observed in $B, V$ and $R$ bands, while the two adjacent fields (dashed contours) were observed only in $V$ band. Circles mark the regions analysed in Sect. 6.1. North is up and East is on the left. The origin of the coordinates coincides with the cluster center $\left(\alpha_{2000}=013152.7, \delta_{2000}=-133641.9\right)$. A region around a bright star was not considered in the analysis (right bottom corner of central field).

Table 1. Information on the photometric observations.

\begin{tabular}{cccc}
\hline \hline Band & $\begin{array}{c}T_{\text {exp }} \\
\mathrm{ks}\end{array}$ & $\begin{array}{c}\text { Seeing } \\
\operatorname{arcsec}\end{array}$ & $\begin{array}{c}\text { Scale } \\
\operatorname{arcsec} / \mathrm{pxl}\end{array}$ \\
\hline$B$ & 1.8 & 0.80 & 0.267 \\
$V$ & $1.26 \times 3$ & 0.80 & 0.267 \\
$R$ & 0.9 & 0.90 & 0.267 \\
\hline
\end{tabular}

compromise is achieved with an aperture of radius 10 pixels (cf. Howell 1989). For each band, we adopted the following calibration relation:

$M=M^{\prime}+\gamma \cdot C-A \cdot X+Z P$,

where $M$ and $C$ are the magnitudes and colours of the standard stars, $M^{\prime}$ is the instrumental magnitude, $\gamma$ is the coefficient of the colour term, $A$ is the extinction coefficient, $X$ is the airmass and $Z P$ is the zero-point. The quantities $\gamma, A$ and $Z P$ were derived by a least square procedure with the IRAF task FITPARAMS. The results of the photometric calibrations are reported in Table 2. Unless otherwise stated, errors on estimated quantities are given at $68 \%$ confidence level (hereafter c.l.).

As a test we compare the $(B-R, V-R)$ diagram of the Landolt standard stars with that of the stars in the cluster field.
Table 2. Results of the photometric calibration of $B V R$ data.

\begin{tabular}{cccccc}
\hline \hline Band & $C$ & $\gamma$ & $A$ & $Z P$ & $\sigma$ \\
\hline$B$ & $B-V$ & $-0.037 \pm 0.008$ & $0.322 \pm 0.010$ & $24.885 \pm 0.015$ & 0.016 \\
$V$ & $B-V$ & $0.035 \pm 0.009$ & $0.204 \pm 0.013$ & $25.277 \pm 0.021$ & 0.020 \\
$R$ & $V-R$ & $0.010 \pm 0.017$ & $0.145 \pm 0.014$ & $25.346 \pm 0.023$ & 0.026 \\
\hline
\end{tabular}

We measured the magnitude of these stars by using the software SExtractor (Bertin \& Arnouts 1996). We verified that the observed distribution of stars in our images matches that of the Landolt stars in the $(B-R, B-V)$ plane, proving the accuracy of the photometric calibration.

\section{Aperture photometry}

For each image, a photometric catalog was derived by using the software SExtractor (Bertin \& Arnouts 1996). We measured magnitudes within a fixed aperture of 5.0", corresponding to $\sim 17 \mathrm{kpc}$ at $z=0.209$, and Kron magnitudes (Kron 1980 ), for which we used an adaptive aperture with diameter $a \cdot r_{\mathrm{K}}$, where $r_{\mathrm{K}}$ is the Kron radius and $a$ is a constant. We chose $a=2.5$, yielding $\sim 94 \%$ of the total source flux within the adaptive aperture (Bertin \& Arnouts 1996). The measured magnitudes were corrected for galactic extinction following Schlegel et al. (1998). The uncertainties on the magnitudes were obtained by adding in quadrature both the uncertainties estimated by SExtractor and the uncertainties on the photometric calibrations.

The completeness magnitudes were derived following the method of Garilli et al. (1999), as shown in Fig. 2. We estimated the completeness magnitude as the magnitude where galaxies start to be lost because they are fainter than the brightness threshold in the detection cell. The completeness magnitudes are $B_{\mathrm{C}}=22.8\left(N_{\mathrm{gal}}=339\right), V_{\mathrm{C}}=22.5\left(N_{\mathrm{gal}}=1078\right)$, and $R_{\mathrm{C}}=22.0\left(N_{\mathrm{gal}}=679\right)$.

In order to derive the LF we considered the objects brighter than the completeness limit and adopted the Kron magnitude, since this is the best estimate of the total magnitude. The star/galaxy classification was based on the SExtractor stellar index $(S G)$, defining as stars the sources with $S G \geq 0.98$.

The catalogs for the central region of the cluster were cross-correlated by using the IRAF task XYXYMATCH. For each object, colours were derived within the fixed aperture.

\section{Luminosity functions}

In order to measure the cluster LF in each band we used all the galaxy photometric data up to the completeness magnitude, and removed the interlopers by statistically subtracting the background contamination. We used galaxy counts in $B$, $V$ and $R$ bands from the ESO-Sculptor Survey (Arnouts et al. 1997; de Lapparent et al. 2003) kindly provided to us by V. de Lapparent. These data cover an area of $\sim 729 \mathrm{arcmin}^{2}$ observed with EMMI instrument. The data reduction was performed by using the procedures similar to those adopted in 


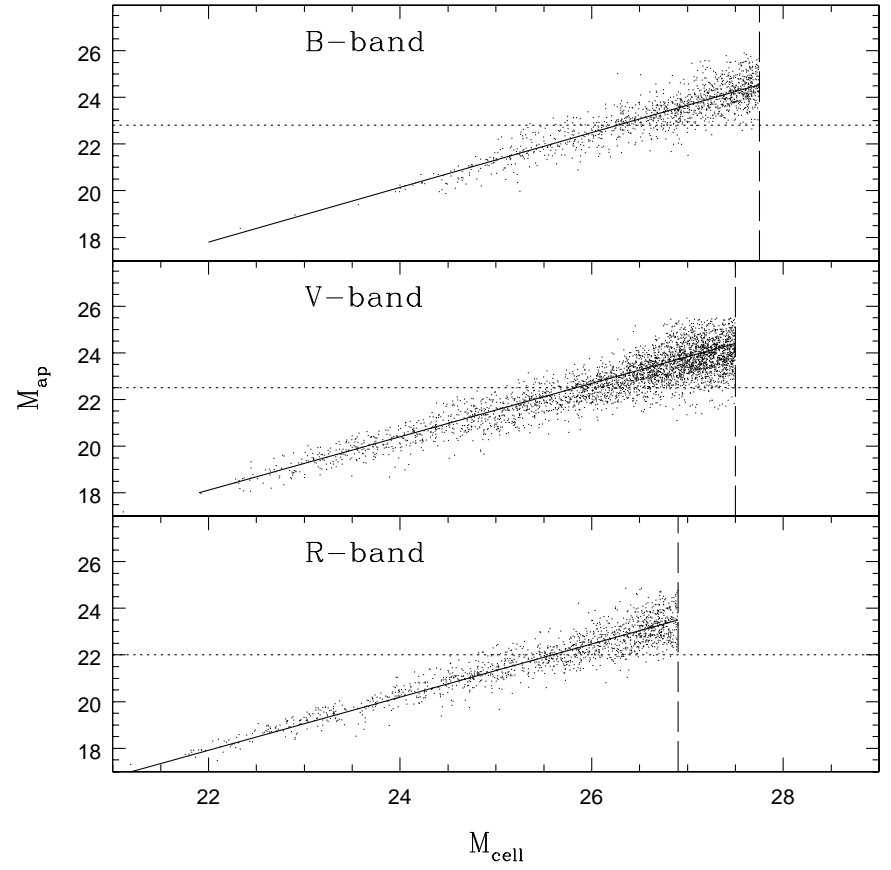

Fig. 2. Completeness magnitudes of the $B-, V-$, and $R$-band images are estimated by comparing magnitudes in the fixed aperture $\left(M_{\mathrm{ap}}\right)$ and in the detection cell $\left(M_{\text {cell }}\right)$. The dotted lines represent the completeness limits, the dashed lines mark the limits in the detection cell, and solid lines are the linear relations between $M_{\text {ap }}$ and $M_{\text {cell }}$.

the present work. In particular, the photometric catalog was obtained by SExtractor and the total magnitudes were estimated from the Kron magnitudes defined by adopting the same aperture.

We assumed Poisson statistics for the background and cluster field galaxy counts. The errors on the cluster LFs were computed by adding in quadrature Poissonian fluctuations. Since the field of view of our observations is $\sim 10$ times smaller than the area covered by the background counts, the errors on the cluster LFs are dominated by Poissonian errors of cluster counts.

We derived the LFs for the central field (see Fig. 1) in the $B, V$ and $R$ bands by fitting the galaxies counts with a single Schechter function. In $V$ and $R$ bands the fits were also computed with the sum of two Schechter functions in order to describe the bright and the faint populations (Sect. 5.1). We compared the LFs with counts obtained selecting red sequence galaxies (Sect. 5.2). All the fit parameters and the $\chi^{2}$ statistics, are listed in Table 3.

\subsection{Multiband analysis}

Figure 3 (solid lines) shows the LFs in the $B, V$ and $R$ bands for the central cluster region, obtained by a weighted parametric fit of the Schechter function to the statistically backgroundsubtracted galaxy counts (filled circles). The parameters of the fit are: $B^{*}=20.06, \alpha_{B}=-1.26, V^{*}=18.29, \alpha_{V}=-1.27$, $R^{*}=17.78, \alpha_{R}=-1.20$. We evaluated the quality of the fits by means of the $\chi^{2}$ statistics (see Table 3). The single Schechter function gives a fair representation of the global distribution of the data, that is the single Schechter fit cannot be rejected in any band even at the $10 \%$ c.l.

On the other hand, there is indication of a dip in the distribution at $V \sim 21.0$ and $R \sim 20.5$. According to the fitted single Schechter function, there should be 121 and 125 galaxies in the range $V=20.5-21.5$ and $R=20.0-21.0$ respectively, whereas in our counts we find $\sim 92 \pm 10$ and $\sim 105 \pm 10$ galaxies. If we define the dip amplitude as:

$A=\frac{N_{\mathrm{e}}-N_{\mathrm{o}}}{N_{\mathrm{e}}}$,

where $N_{\mathrm{e}}$ and $N_{\mathrm{o}}$ are the expected and observed number of galaxies in the dip magnitude range, we obtain in $V(R)$ band $A=24 \pm 8 \%(16 \pm 8 \%)$. The position of dips are: $M_{V} \sim-19.5$ and $M_{R} \sim-19.8$. These values were obtained by converting apparent into absolute magnitudes using k-corrections for earlytype galaxies from Poggianti (1997). The $B$-band data are not deep enough to sample the dip position.

The presence of a dip was discussed in the literature by considering the properties of the TSLFs (e.g., Binggeli et al. 1988) in nearby clusters. In a study of the Virgo cluster, Sandage et al. (1985) showed the presence of two distinct classes of galaxies (normal and dwarfs), with different dynamical and luminosity evolution. Since the bright-end of the LF is well studied, we can use a priori the information about the LF shape for bright galaxies to fit our data with two different Schechter functions, representing bright and faint galaxies. We assumed a Schechter model with $R_{\text {bright }}^{*}=18.0$ and $\alpha_{R \text {,bright }}=-1.0$ (from Näslund et al. 2000 results, scaled according to the adopted cosmology). Using the $V-R$ colour term as derived from Eq. (4), we also fix $V_{\text {bright }}^{*}=18.7$ and $\alpha_{V \text {,bright }}=-1.0$.

Figure 4 shows the cluster LFs in $V$ and $R$ bands modelled with the two Schechter functions. The fit procedures yields $V_{\text {faint }}^{*}=21.75, R_{\text {faint }}^{*}=21.12$. For the slope of the faint galaxies function the $V$ - and $R$-band data provide poor constraints, so that we have only an indication of a steep faintend. According to the $\chi^{2}$ statistics (see Table 3), combining two Schechter functions for bright and faint galaxies the quality of the fit increases both in $V$ and $R$ bands.

\subsection{Galaxies on the red sequence}

We obtained the Colour-Magnitude (CM) relation by fitting the photometric data of the spectroscopically confirmed cluster members (see Paper I) with a biweight algorithm (Beers et al. 1990):

$(V-R)_{\mathrm{CM}}=-0.023 \cdot R+1.117$.

By using Eq. (4), we defined as sequence galaxies the sources lying in the region inside the curves:

$(V-R)_{\mathrm{seq}}=(V-R)_{\mathrm{CM}} \pm\left(\sqrt{\sigma_{V}^{2}+\sigma_{R}^{2}}+0.05\right)$,

where we took into account the photometric uncertainty at $1 \sigma$ both on the $V\left(\sigma_{V}\right)$ and on the $R$ magnitude $\left(\sigma_{R}\right)$ as well as the intrinsic dispersion of the CM relation (Moretti et al. 1999). In Fig. 5 the red sequence galaxies are marked with filled circles. 
Table 3. Fits to the Luminosity Functions. Errors on the $M^{*}$ and $\alpha$ parameters can be obtained by the confidence contours shown in Fig. 3 .

\begin{tabular}{c|cccc|cccc}
\hline \hline Band & \multicolumn{5}{|c|}{ Single Schechter function } & \multicolumn{5}{c}{ Two Schechter functions $^{a}$} \\
\hline & $M^{*}$ & $\alpha$ & $\chi_{v}^{2 b}$ & $P\left(\chi^{2}>\chi_{v}^{2}\right)$ & $M_{\text {faint }}^{*}$ & $\alpha_{\text {faint }}$ & $\chi_{v}^{2}$ & $P\left(\chi^{2}>\chi_{v}^{2}\right)$ \\
\hline$B$ & -21.03 & -1.26 & 0.96 & $46 \%$ & & & & \\
$V^{c}$ & -22.03 & -1.25 & 1.04 & $40 \%$ & & & & \\
$V$ & -22.18 & -1.27 & 1.46 & $15 \%$ & -18.72 & -2.00 & 1.09 & $37 \%$ \\
$R$ & -22.48 & -1.20 & 1.24 & $27 \%$ & -19.14 & -1.24 & 1.06 & $39 \%$ \\
\hline \multicolumn{8}{c}{ Galaxies on the red sequence } \\
\hline$V$ & -22.60 & -1.33 & 2.10 & $2 \%$ & -18.68 & -2.19 & 1.68 & $9 \%$ \\
$R$ & -22.76 & -1.27 & 1.30 & $23 \%$ & -19.15 & -1.39 & 1.09 & $37 \%$ \\
\hline
\end{tabular}

${ }^{a}$ The bright-end LF is fixed (see text).

${ }^{b}$ The reduced $\chi^{2}$.

${ }^{c}$ Total observed field (see Sect. 6).

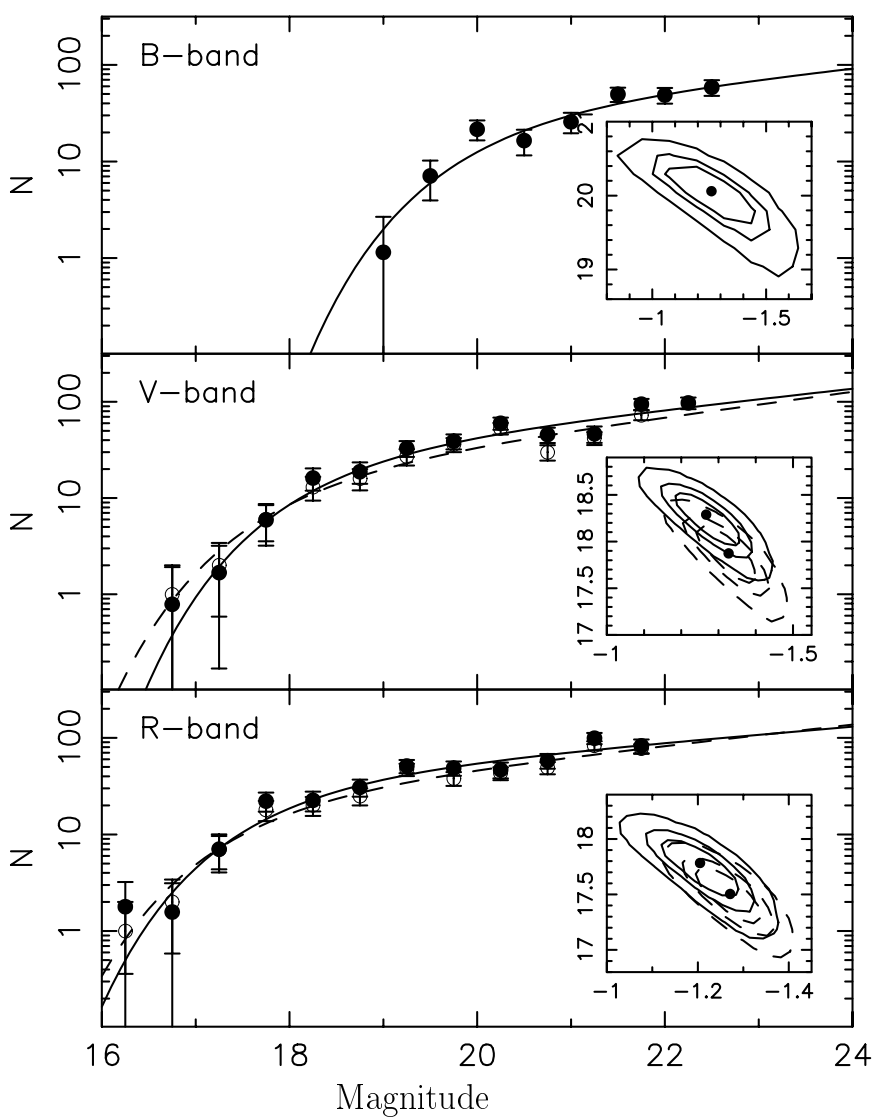

Fig. 3. Luminosity function in the $B, V$ and $R$ bands in the central field of $9.2^{\prime} \times 8.6^{\prime}$. In $V$ and $R$ bands filled circles represent counts obtained from the photometric catalog with a statistical background subtraction, open circles are the counts of galaxies belonging to the red sequence of the CM relation (see Sect. 5.2). Solid and dashed lines are the Schechter fits to the filled and open circles respectively. In the small panels the 1,2 and $3 \sigma$ c.l. of the best-fit parameters $\alpha$ and $M^{*}$ are shown.

By selecting galaxies on the CMR (within the observed scatter), the background contamination is expected to be negligible. So we can directly compare galaxy counts (open circles in Fig. 3) with those derived in Sect. 5.1 (filled circles in Fig. 3).
In both $V$ and $R$ bands the counts obtained with the two different approaches are very similar.

Figure 3 (dashed lines) shows the LFs in the $V$ and $R$ bands for the central cluster region, obtained by a weighted parametric fit of the Schechter function to the red galaxy counts (open circles). The parameters of the fit are: $V^{*}=17.87, \alpha_{V}=-1.33$, $R^{*}=17.50, \alpha_{R}=-1.27$. According to the $\chi^{2}$ statistics (see Table 3) we can reject the fit with a single Schechter function in the $V$ band at $98 \%$ c.l. At the same time, the dip amplitude in the $V$ band, $A=26 \pm 9 \%$, is unchanged respect to the case of the global LF (Sect. 5.1). It turns out that, independently from the fitted Schechter functions, the ratio $(\sim 60 \%)$ of observed counts in the bins inside the dip and in the bins adjacent to the dip regions is the same for both galaxy samples, while the overall distribution of red galaxies cannot be described with a single Schechter function. According to the $\chi^{2}$ statistics (see Table 3), combining two Schechter functions the quality of the fit increases both in $V$ and $R$ bands, and becomes acceptable also in the $V$ band.

\section{Luminosity segregation}

The data in the $V$ band, covering an area of $\sim 160 \mathrm{arcmin}^{2}$, corresponding to a circular region with equivalent radius $0.6 R_{\text {vir }}$ $\left(R_{\mathrm{vir}}=2.5 h_{70}^{-1} \mathrm{Mpc}\right.$; see Paper I), allow to study the environmental dependence of LF and the spatial distribution of galaxies as a function of the clustercentric distance.

Figure 6 (upper panel) shows the $V$-band LF in the whole observed area, modelled by using a weighted parametric fit to a single Schechter function, with best fit values $V^{*}=18.45$ $\left(M_{V}^{*}=-22.03\right)$ and $\alpha_{V}=-1.25$. The LF shape is very similar to that obtained in the central field (Fig. 3) and also in this case the Schechter function overestimates the observed counts in the range $V=20.5-21.5$. The dip amplitude is $A=14 \pm 7 \%$.

\subsection{The $L F$ in different cluster regions}

In order to compare the abundances of galaxies of various luminosities in different regions we computed the LFs in three areas 
Table 4. Best fit value of the LFs measured in three regions around the center of the cluster.

\begin{tabular}{cccccc}
\hline \hline Region & $\operatorname{Area}\left(h_{70}^{-2} \mathrm{Mpc}^{2}\right)$ & $V^{*}$ & Luminosity ratio & $\chi_{v}^{2}$ & $P\left(\chi^{2}>\chi_{v}^{2}\right)$ \\
\hline A & 0.50 & $18.09 \pm 0.73$ & $12.7 \pm 3.0$ & 1.12 & $35 \%$ \\
B & 1.00 & $18.49 \pm 0.44$ & $6.9 \pm 1.4$ & 1.08 & $37 \%$ \\
C & 1.00 & $18.88 \pm 0.43$ & $6.8 \pm 2.3$ & 0.85 & $56 \%$ \\
\hline
\end{tabular}

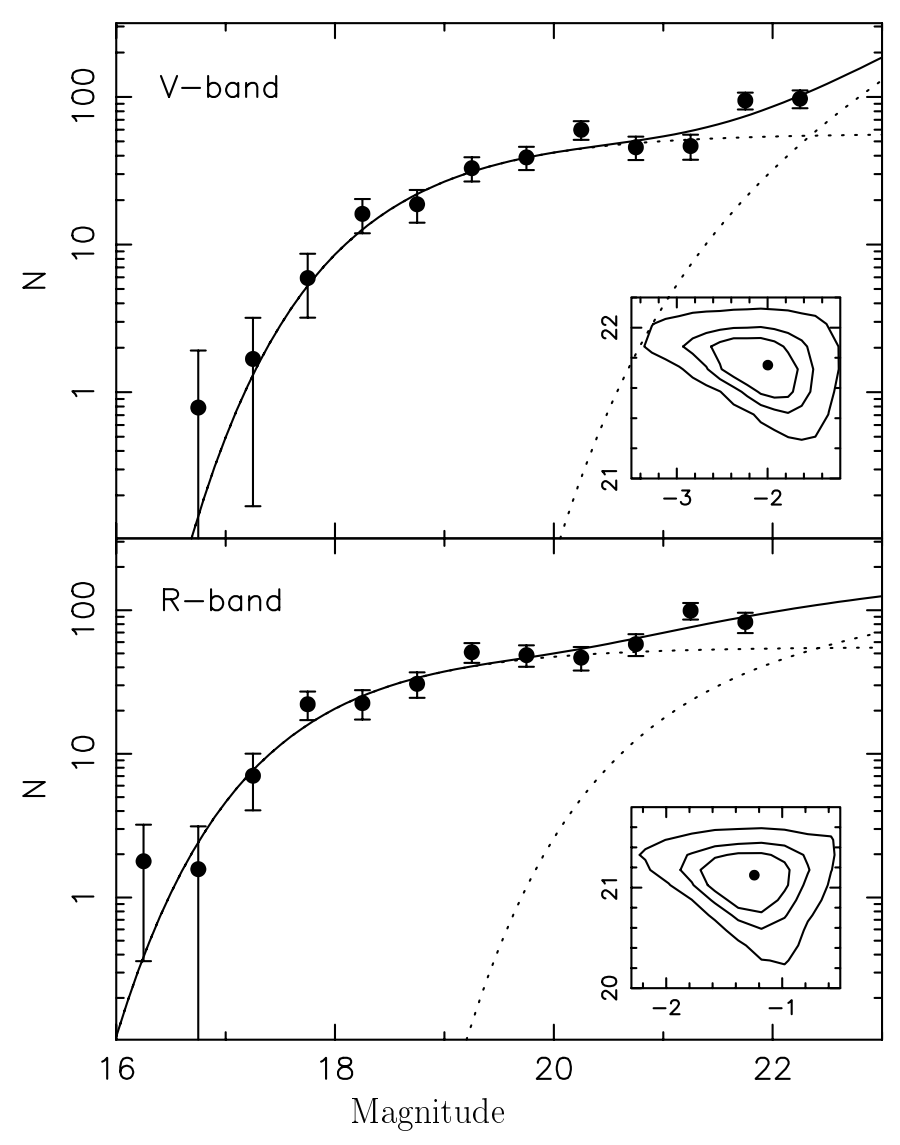

Fig. 4. Luminosity function in the $V$ and $R$ bands in the central field of $9.2^{\prime} \times 8.6^{\prime}$. The dashed lines represent the Schechter functions for bright and faint galaxies separately (see text). The continuous line represents the sum of the two functions. In the small panels the 1, 2 and $3 \sigma$ c.l. of the best-fit parameters $\alpha$ and $M^{*}$ are shown.

at different distances from the center ${ }^{2}$. First we considered the cluster counts in an area of $0.5 h_{70}^{-2} \mathrm{Mpc}^{2}$ around the center of the cluster (region A), and then in two concentric circular rings around the first central region, each in an area of $1.0 h_{70}^{-2} \mathrm{Mpc}^{2}$, respectively at $\sim 2^{\prime}$ and $\sim 4.7^{\prime}$ from the center (region B and C). We fitted the counts using a single Schechter function with $\alpha$ fixed at the best fit value obtained from the LF computed over the whole observed field. Table 4 reports the relative fit values in the different regions and Fig. 6 shows the fitted functions. We also measured the luminosity weighted ratio of the number of objects brighter and fainter than the $V=21.0$ (Table 4 ), that

\footnotetext{
2 The center of the cluster was derived by a two-dimensional adaptive kernel technique from the spectroscopically confirmed cluster members (for details see Paper I) and coincides with the $\mathrm{cD}$ position.
}

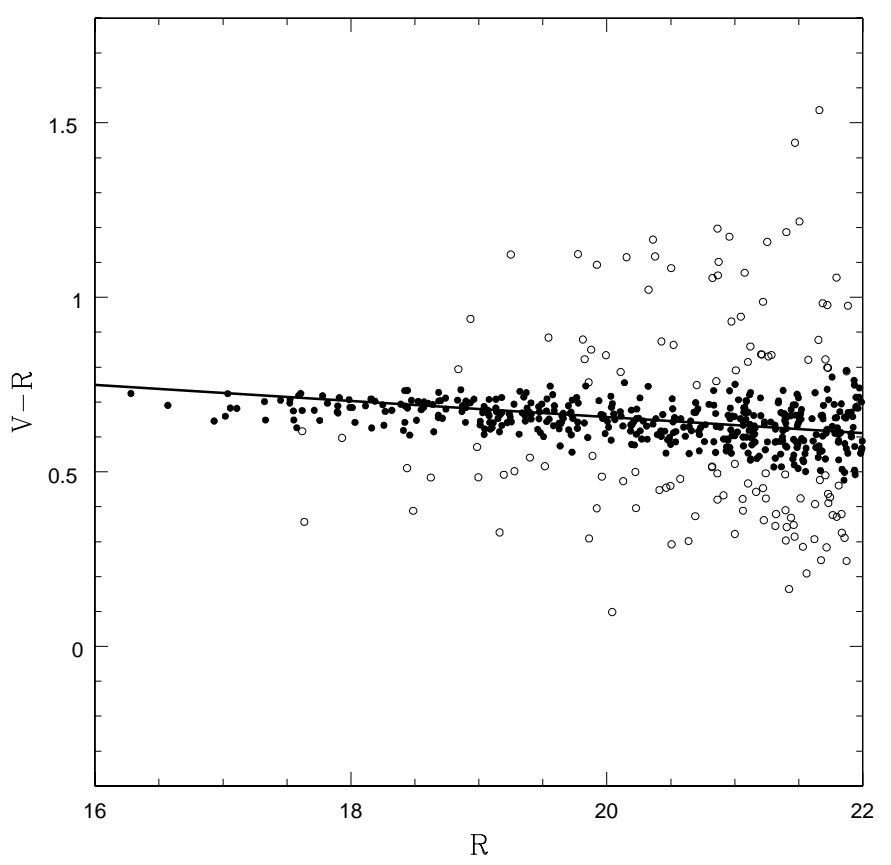

Fig. 5. $V-R$ vs. $R$ CM diagram for all the galaxies within the completeness limit in the central field of ABCG 209. Galaxies of the red sequence (see solid line) are plotted as filled circles. The solid line defines the CM sequence for spectroscopically confirmed cluster members.

is the magnitude where the dip occurs. This luminosity ratio increases from region $\mathrm{B}$ to the center by a factor $1.8 \pm 0.6$, indicating a significant luminosity segregation, whereas the ratio does not vary from the region $\mathrm{B}$ to the region $\mathrm{C}$.

\subsection{Spatial distribution of galaxies}

In Fig. 7 (left panel) the number density radial profile is shown, i.e. the number of galaxies measured in concentric rings around the center. It has been computed for the whole galaxy population (open circles) and for galaxies brighter (filled circles) and fainter (triangles) than $V=21.0$, the magnitude where the luminosity distribution shows the distinctive upturn. The number of faint galaxies is determined up to the completeness limit. The densities are statistically background-subtracted by using background galaxy density measured in the ESOSculptor Survey (Arnouts et al. 1997). Errors are assumed to be Poissonian.

The radial profile of all galaxies is centrally peaked, showing the highest gradient for bright galaxies (Fig. 7, left panel). Indeed, the bright galaxy density decreases by a factor $\sim 5.4$ 


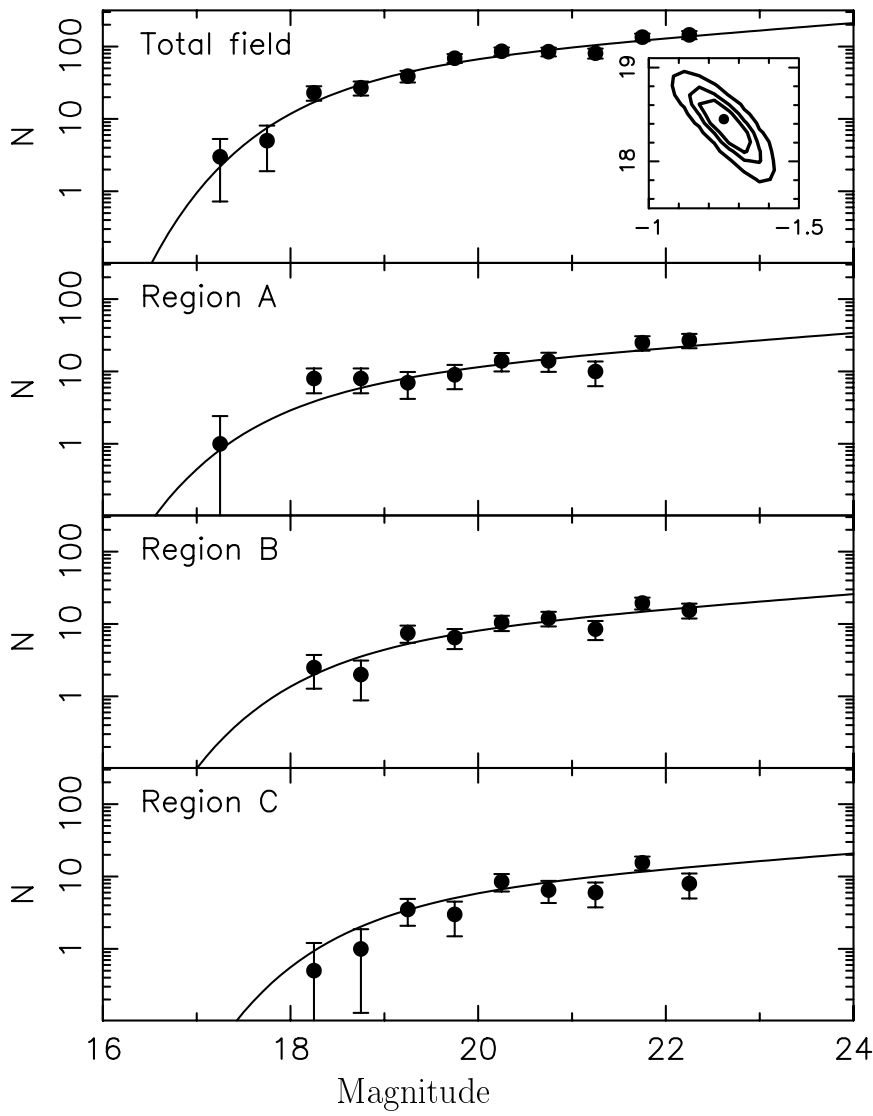

Fig. 6. Luminosity function in the $V$ band. In the upper panel are shown the LF in the whole observed area $\left(160 \operatorname{arcmin}^{2}\right)$ and the 1, 2 and $3 \sigma$ c.l. of the best-fit parameters $\alpha$ and $M^{*}$ (small panel). The continuous line represents the fitted Schechter functions. In the other panels the LFs in the three cluster regions (see text) are shown. Continuous lines are the fit of the Schechter functions obtained by fixing the faint-end slope at the best fit value of the LF fitted on the whole observed area.

between the first and the second bin, to be compared with a decrease of a factor $\sim 1.4$ for faint galaxies and of a factor $\sim 2.6$ when all galaxies are considered. For faint galaxies $(V>21.0)$, the distribution decreases slowly with clustercentric distance.

Figure 7 (right panel) shows the bright-to-faint ratio (BFR) as a function of the clustercentric distance. The BFR shows a maximum at the center, where the number of bright galaxies is two times the number of faint galaxies, and decreases rapidly between the first and the second bin. Outside the central region of the cluster ( $r>1$ arcmin) the BFR distribution is flat and there are roughly two faint galaxies for each bright galaxy.

\section{Summary and discussion}

The analysis performed on ABCG 209 can be summarized by the following results:

1) the fit with a single Schechter function cannot be rejected in any band; at the same time, the luminosity distributions are better described by a sum of two Schechter functions for bright and for faint galaxies respectively;
2) there is an indication of a dip in $V$ - and $R$-band LFs, and this features is also present when considering only galaxies that lie on the red sequence;

3) the faint-end slope seems to be steeper going from redder to bluer wavelengths;

4) there is a luminosity segregation, in the sense that the $V^{*}$ value increases from the center to the outer regions and the luminosity ratio of bright-to-faint galaxies decreases by a factor $\sim 2$ from the center to outer regions, whereas the number ratio decreases by a factor $\sim 4$.

The presence of a dip was found in the LF of many clusters. In Table 5 we reported the positions of the dips for some of these clusters with $0.0<z<0.3$ in $R$-band absolute magnitudes (according to the adopted cosmology). The literature data were transformed to $R$-band absolute magnitudes by using kand evolutionary correction, and average colours for early-type galaxies of Poggianti (1997).

Dips are found at comparable absolute magnitudes, in different clusters, suggesting that these clusters may have similar galaxy population. However, the dips have not necessarily the same shape: the dips found in the the LF of Shapley 8 and Coma are broader than those found in Virgo, ABCG 963, and ABCG 85. As already mentioned in the introduction, this can be explained with the hypothesis of an universal TSLF (Binggeli et al. 1988), whereas the different relative abundances of galaxy types, induced by cluster-related processes, are at the origin of the different dip shapes (see also Andreon 1998).

Moreover, as shown in Table 5, there is no correlation between the dip position and the redshift, suggesting little or no evolutionary effect in the dip, in agreement with Näslund et al. (2000), which directly compare the LFs of Coma, ABCG 963 and MS 2255. They detected no qualitative difference between nearby and distant clusters.

Studying the $R$-band LFs for a photometric sample of 10 clusters at different redshifts and with different richness classes, Yagi et al. (2002) demonstrated that the dips seen in the LFs are almost entirely due to $r^{1 / 4}$-like galaxies. This evidence is in agreement with those shown in Fig. 3 (open circles). In fact by comparing the counts of red sequence galaxies with those obtained with a statistical background subtraction, the dip in $V$ band appears more pronounced.

Environmental effects can be at the origin of the different positions of the dips seen in different clusters, since the transition from the bright-dominated to the faint-dominated parts of the LF can occur at different magnitudes in different environment. The cluster morphological mix and the morphologydensity relation (Dressler 1980) should give rise to LFs with different shape when subdividing a sample galaxies respect to the cluster environments.

The effect of the environment can be seen in ABCG 209 by comparing the shape of the LF in $V$ band in different regions around the cluster center (see Fig. 6 and Sect. 6.1). The fitted $M^{*}$ value is shifted toward fainter magnitudes going from the inner $(\mathrm{A})$ to the outer $(\mathrm{C})$ region and the luminosity ratio of bright-to-faint galaxies decreases by a factor $\sim 2$, indicating a luminosity segregation. 

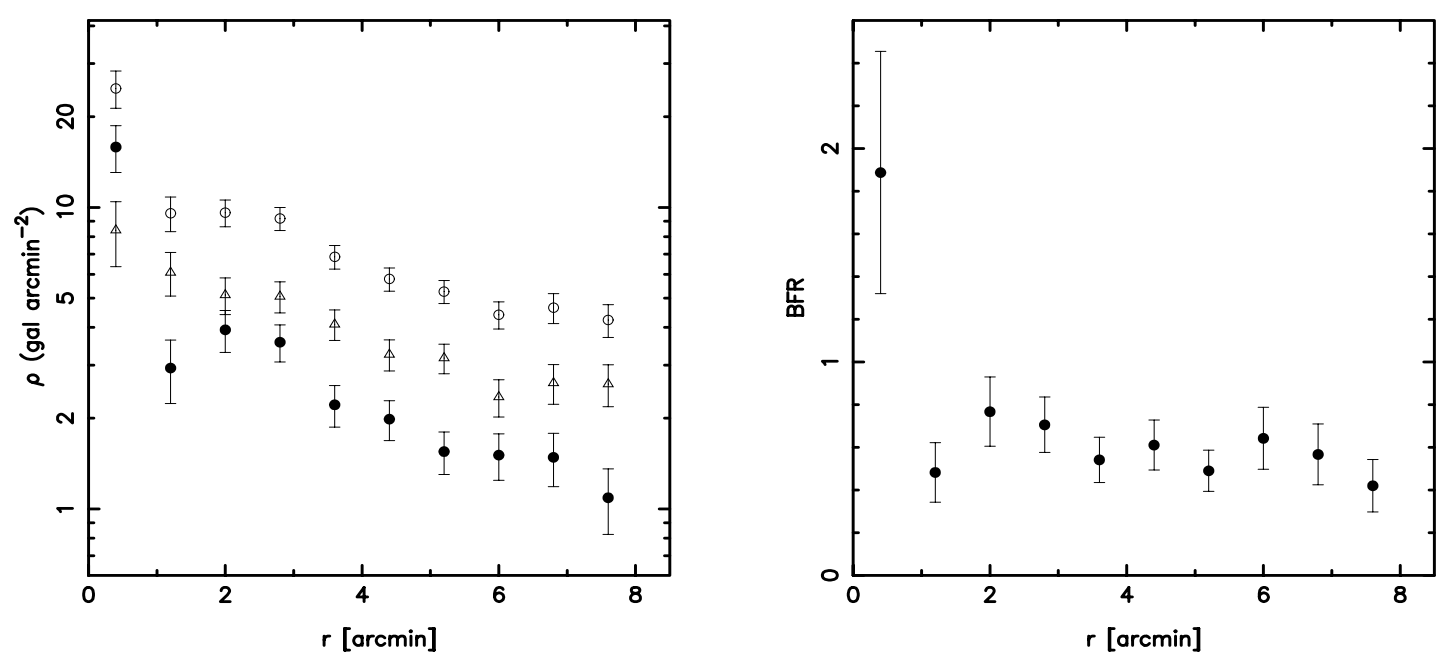

Fig. 7. Background-subtracted radial profile of ABCG 209 is shown in the left panel. Open circles represent the distribution of all galaxies in the cluster, filled circles are bright galaxies $\left(M_{V}^{*} \leq-19.5\right)$ and open triangles are faint galaxies $\left(M_{V}^{*}>-19.5\right)$. The number of bright galaxies in the first and in the second bin is $\sim 32$ and $\sim 17$ respectively, while the number of faint galaxies is $\sim 15$ and $\sim 30$. The bright-to-faint ratio as a function of the clustercentric distance is shown in the right panel.

Table 5. Compilation of dip positions in the restframe $R$ band in cluster LFs from literature. Data were transformed to $R$-band absolute magnitudes by using $\mathrm{k}$ - and evolutionary correction, and average colours for early-type galaxies of Poggianti (1997).

\begin{tabular}{cccc}
\hline \hline Cluster name & Redshift & Dip position & Reference \\
\hline Virgo & 0.0040 & -18.9 & Sandage et al. (1985) \\
ABCG 194 & 0.018 & -19.8 & Yagi et al. (2002) \\
Coma & 0.0232 & -19.8 & Biviano et al. (1995) \\
ABCG 496 & 0.0331 & -19.8 & Durret et al. (2000) \\
ABCG 2063 & 0.035 & -19.3 & Yagi et al. (2002) \\
ABCG 576 & 0.038 & -18.6 & Mohr et al. (1996) \\
Shapley8 & 0.0482 & -19.5 & Metcalfe et al. (1994) \\
ABCG 754 & 0.053 & -19.3 & Yagi et al. (2002) \\
ABCG 85(z) ${ }^{a}$ & 0.0555 & -19.9 & Durret et al. (1999) \\
ABCG 85 & 0.0555 & -19.3 & Durret et al. (1999) \\
ABCG 2670 & 0.076 & -19.3 & Yagi et al. (2002) \\
ABCG 1689 & 0.181 & -18.7 & Wilson et al. (1997) \\
ABCG 665 & 0.182 & -18.7 & Wilson et al. (1997) \\
ABCG 963 & 0.206 & -19.0 & Driver et al. (1994) \\
ABCG 209 & 0.209 & -19.6 & This paper \\
MS 2255.7 + 2039 & 0.288 & -19.6 & Näslund et al. (2000) \\
\hline
\end{tabular}

${ }^{a}$ LF fitted only on the spectroscopically confirmed cluster galaxies.

The bright galaxies are markedly segregated in the inner $0.2 h_{70}^{-1}$, around the $\mathrm{cD}$ galaxy. This suggest that bright galaxies could trace the remnant of the core-halo structure of a pre-merging clump.

Although ABCG 209 is a cD-like cluster, with cD galaxy located in the center of a main X-ray peak, it shows an elongation and asymmetry in the galaxy distribution (Paper I).
Moreover, the faint-end slope turns out to be $\alpha<-1$ at more than $3 \sigma$ c.l. in both $V$ and $R$ bands, thus reconciling the asymmetric properties of $\mathrm{X}$-ray emission with the non flat-LF shape of irregular systems as found by Lopez-Cruz et al. (1997).

These results allow to discriminate between the two possible formation scenarios suggested by the dynamical analysis (Paper I). We conclude that ABCG 209 is an evolved cluster, resulting from the merger of two or more sub-clusters, while the elongation and asymmetry of the galaxy distribution (of the X-ray emission) and the shape of the LFs show that ABCG 209 is not yet a fully relaxed system.

Our analysis is in agreement with the existence of i) an universal LF for bright galaxies, which is well described by a flat Schechter function, and ii) a steep Schechter function for faint galaxies. A definitive conclusion regarding the faint-end slope of the LF needs deeper photometry able to sample the luminosity distribution of dwarf galaxies.

Acknowledgements. We thank Valerie de Lapparent who provided us with the galaxy counts used to derive the cluster LFs. A. M. thanks Massimo Capaccioli for the hospitality at the Osservatorio Astronomico di Capodimonte, and Francesca Matteucci for support during this work. This work has been partially supported by the Italian Ministry of Education, University, and Research (MIUR) grant COFIN2001028932: clusters and groups of galaxies, the interplay of dark and baryonic matter, and by the Italian Space Agency (ASI).

\section{References}

Abell, G. O., Corwin, H. G. Jr., \& Olowin, R. P. 1989, ApJS, 70, 1

Andreon, S. 1998, A\&A, 336, 98

Andreon, S. 2001, ApJ, 547, 623

Arnouts, S., de Lapparent, V., Mathez, G., et al. 1997, A\&AS, 124, 163

Beers, T. C., Flynn, K., \& Gebhardt, K. 1990, AJ, 100, 32

Bertin, E., \& Arnouts, S. 1996, A\&AS, 331, 439

Binggeli, B., Sandage, A., \& Tammann, G. A. 1988, ARA\&A, 26, 509

Biviano, A., Durret, F., Gerbal, D., et al. 1995, A\&A, 297, 610 
Colless, M. 1989, MNRAS, 237, 799

de Lapparent, V., Galas, G., Bardelli, S., \& Arnouts, S. 2003, A\&A, 404, 831

De Propris, R., Pritchet, C. J., Harris, W. E., \& McClure, R. D. 1995, ApJ, 450, 534

Dressler, A. 1980, ApJ, 236, 351

Driver, S. P., Phillips, S., Davies, J. I., Morgan, I., \& Disney, M. J. 1994, MNRAS, 268, 393

Durret, F., Gerbal, D., Lobo, C., \& Pichon, C. 1999, A\&A, 343, 774

Durret, F., Adami, C., Gerbal, D., \& Pislar, V. 2000, A\&A, 356, 815

Ebeling, H., Voges, W., Böhringer, H., et al. 1996, MNRAS, 281, 799

Efstathiou, G., Ellis, R. S., \& Peterson, B. A. 1988, MNRAS, 232, 431

Feretti, L. 2002, in The Universe at Low Radio Frequences, ed.

A. Pramesh Rao, G. Swarup, \& Gopal-Krishna (Kluwer Academic Publishers), S-199 available December 2002 [astro-ph/0006379]

Fetisova, T. S. 1981, Astron. Zh., 58, 1137

Garilli, B., Maccagni, D., \& Andreon, S. 1999, A\&A, 342, 408

Giovannini, G., Tordi, M., \& Feretti, L. 1999, New Astron., 4, 141

Howell, S. B. 1989, PASP, 101, 616

Kristian, J., Sandage, A., \& Westphal, J. A. 1978, ApJ, 221, 383

Kron, R. G. 1980, ApJS, 43, 305

Landolt, A. U. 1992, AJ, 104, 340

Lopez-Cruz, O., Yee, H. K. C., Brown, J. P., et al. 1997, ApJ, 475, L97

Loveday, J., Peterson, B. A., Efstathiou, G., \& Maddox, S. J. 1992, ApJ, 390, 338

Lugger, P. M. 1986, ApJ, 303, 555
Lumsden, S. L., Collins, C. A., Nichol, R. C., Eke, V. R., \& Guzzo, L. 1997, MNRAS, 290, 119

Metcalfe, N., Godwin, J. G., \& Peach, J. V. 1994, MNRAS, 267, 431

Mercurio, A., Girardi, M., Boschin, W., Merluzzi, P., \& Busarello, G. 2003, A\&A, 397, 431

Mohr, J. J., Geller, M. J., Fabricant, D. G., et al. 1996, ApJ, 470, 720

Molinari, E., Chincarini, G., Moretti, A., \& De Grandi, S. 1998, A\&A, 338,874

Moretti, A., Molinari, E., Chincarini, G., \& De Grandi, S. 1999, A\&AS, 140, 155

Näslund, M., Fransson, \& Huldtgren, M. 2000, A\&A, 356, 435

Oelmer, A. 1974, ApJ, 194, 1

Poggianti, B. M. 1997, A\&AS, 122, 399

Press, W. J., \& Schechter, P. 1974, ApJ, 187, 425

Rizza, E., Burns, J. O., Ledlow, M. J., et al. 1998, MNRAS, 301, 328

Sandage, A., Binggeli, B., \& Tamman, G. A. 1985, AJ, 90, 1759

Schechter, P. 1976, ApJ, 203, 297

Schlegel, D. J., Finkbeiner, D. P., \& Davis, M. 1998, ApJ, 500, 525

Trentham, N. 1997, MNRAS, 286, 133

Trentham, N. 1998, MNRAS, 294, 193

Trentham, N., \& Hodgkin, S. 2002, MNRAS, 333, 423

Valotto, C. A., Nicotra, M. A., Muriel, H., \& Lambas, D. G. 1997, ApJ, 479, 90

Wilkinson, A., \& Oke, J. B. 1978, ApJ, 220, 376

Wilson, G., Smail, I., Ellis, R. S., \& Couch, W. J. 1997, MNRAS, 284, 915

Yagi, M., Kashikawa, N., Sekiguchi, M., et al. 2002, AJ, 123, 87 